\title{
Metapesquisa No Campo Da Educação Estatística Com Foco Na Educação \\ De Jovens E Adultos
}

\author{
Meta-Research In The Field Of Education Statistical Focus On Youth And Adult \\ Education
}

\author{
Reinaldo Feio Lima* \\ Universidade Federal do Sul e Sudeste do Pará - (UNIFESSPA) \\ Ilvanete dos Santos de Souza** \\ Universidade Federal da Bahia - (UFBA) \\ Américo Junior Nunes da Silva*** \\ Universidade do Estado da Bahia - (UNEB)
}

\begin{abstract}
Resumo
O presente artigo tem como propósito identificar e compreender como se apresentam a produção da pesquisa em Educação Estatística que tem como foco o ensino aprendizagem na Educação de Jovens e Adultos. Metodologicamente trata-se de uma pesquisa de abordagem qualitativa interpretativa e que se utilizou dos princípios de uma metapesquisa a partir do levantamento dos trabalhos publicados nos anais de 04 eventos da área da Educação Matemática: i) Seminário Internacional de Pesquisa em Educação Matemática (SIPEM); ii) Encontro Nacional de Educação Matemática (ENEM); iii) Congresso Internacional de Ensino de Matemática (CIEM); e iv) Simpósio Internacional de Pesquisa em Educação Matemática (SIPEMAT). Após leitura atenciosa do título, resumo e palavras-chave encontramos um universo de 172 trabalhos no campo da Educação Estatística. Desses, apenas 10 (dez) trabalhos contemplaram a Educação de Jovens e Adultos e passaram a constituir nosso corpus de pesquisa. Foram definidas seis categorias a priori para análise: (a) sobre as instituições; (b) sobre autorias; (c) método e instrumentos de produção de dados; (d) sobre os aportes teóricos; (e) conteúdo abordado e; (f) o papel do estudante da EJA na pesquisa. Os resultados apontam que os textos analisados apresentam uma variedade de temáticas. Frente a essa compreensão revela-se que a pesquisa em Educação Estatística continua buscando e apresentando argumentos, novas metodologias e propostas didáticas de ensino que justifiquem a sua utilização em sala aula.
\end{abstract}

Palavras-chave: Metapesquisa, Educação Estatística, Educação de Jovens e Adultos.

\begin{abstract}
The purpose of this article is to identify and understand how research that uses Statistical Education as an aid to teaching and learning in Youth and Adult Education is presented. Methodologically, this is a qualitative and interpretive research that uses the principles of a meta-research from the survey of the works published in the annals of 04 events in the area of Mathematics Education, International Seminar

\footnotetext{
* Doutorando em Educação pela UFBA. Professor Assistente da área temática Educação Matemática da Universidade Federal do Sul e Sudeste do Pará (UNIFESSPA), Marabá, PA. E-mail: reinaldo.lima@unifesspa.edu.br

** Doutoranda em Educação pela UFBA. E-mail: ilvanetess@hotmail.com.br

*** Doutor em Educação pela Universidade Federal de São Carlos (UFSCar). Professor da Universidade do Estado da Bahia (Uneb). E-mail: amerjun2005@hotmail.com.br
} 
on Research in Mathematics Education (SIPEM), National Meeting of Mathematics Education (ENEM), International Conference on Teaching Mathematics (CIEM) and the International Symposium on Research in Mathematics Education (SIPEMAT). After careful reading of the title, abstract and keywords, we found a universe of 172 works in the field of Statistical Education. Of these, only 10 (ten) studies contemplated the Education of Youths and Adults began to constitute our corpus of research. Six a priori categories were defined for analysis: (a) on institutions; (b) on authorship; (c) method and instruments of data production; (d) on the theoretical contributions; (e) content addressed and; (f) the role of the EJA student in the research. The results show that the texts analyzed here reveal the variety of themes addressed in these works. Facing this understanding, it is revealed that the research in Statistics Education continues to seek and present arguments, new methodologies and didactic teaching proposals that justify its use in the classroom.

Keywords/Palabras clave: Metapesearch, Statistical Education, Youth and Adult Education.

\section{Introdução}

Nesse artigo teórico são trazidos e discutidos, resumidamente, os resultados de um estudo que se propôs a identificar e compreender como se apresentam as pesquisas realizadas recentemente e que utilizam a Educação Estatística (EE) como auxílio para o ensino e aprendizagem na Educação de Jovens e Adultos (EJA), a fim de analisar que aspectos e dimensões vêm sendo destacados e privilegiados nessas produções, assim como problematizar questões que emergiram a partir das referidas pesquisas.

Para atingir o objetivo proposto realizou-se um mapeamento das publicações acadêmico-científicas feitas no período de 2012 a 2018, tendo como referencial o descritor "Educação Estatística". Este descritor foi utilizado por entender que nos remete às publicações que tem por objetivo discutir as pesquisas voltadas a Educação Estatística. O mapeamento foi realizado em 04 eventos da área de Educação Matemática, a saber: Seminário Internacional de Pesquisa em Educação Matemática (SIPEM), Encontro Nacional de Educação Matemática (ENEM), Congresso Internacional de Ensino de Matemática (CIEM) e o Simpósio Internacional de Pesquisa em Educação Matemática (SIPEMAT). A escolha dos eventos se deu em função da disponibilidade de acesso aos artigos em plataformas de acesso online.

Segundo André (2010), a restrição a um grupo resumido de eventos não acarreta numa grave perda de representatividade, pois a maior parte das pesquisas brasileiras parece ser, de fato, socializadas no âmbito dos eventos com abrangência na Educação Matemática, como os listados no parágrafo anterior. Para isso foi utilizado o procedimento metodológico que consiste na leitura do título, resumo e palavras-chave, fichamento e organização dos trabalhos selecionados.

A escolha dos eventos que comporam o corpus de investigação deste artigo se justifica 
por: a) são eventos muito expressivos para as pesquisas em Educação Matemática; b) esses eventos permitem que os participantes, dentre eles alunos de graduação e pós-graduação, professores da educação básica e pesquisadores se reúnam e compartilhem conhecimentos, experiências e aspirações em relação ao ensino e aprendizagem da Educação Estatística e socialização na comunidade acadêmica. Nessa direção, portanto, torna-se relevante realizar uma metapesquisa com a intensão de construir uma síntese dos dados identificados até o momento em investigações a respeito deste tema (Bicudo \& Paulo, 2011; Cury, 2013; Bicudo \& Miarka, 2015), descrevendo seus principais resultados e sinalizando os novos encaminhamentos advindos dessas produções.

Este contexto descrito possibilita a questão que surge como norteadora da investigação realizada: O que se mostra sobre a pesquisa em Educação Estatística na Educação de Jovens e Adultos publicadas nos anais do SIPEM, ENEM, CIEM e SIPEMAT, entre os anos de 2012 a 2018? O artigo, ora apresentado, organiza-se da seguinte forma: i) seção introdutória em que delineia-se o tema; ii) apresenta-se os procedimentos metodológicos; iii) propõe-se uma apresentação e análise dos dados e iv) versa sobre as considerações finais.

\section{Procedimentos Metodológicos}

Esta pesquisa é de abordagem qualitativa interpretativa (Bogdan \& Biklen, 2010) e tem como objetivo identificar e compreender como se apresentam as pesquisas que utilizam a Educação Estatística como auxílio para o ensino e a aprendizagem na Educação de Jovens e Adultos. Para tal, utilizam-se os princípios de uma metapesquisa, definida por Bicudo e Paulo (2011, p.255), “como pesquisa sobre a pesquisa". Portanto, trata-se de um movimento reflexivo sobre o que foi investigado, sobre como a pesquisa foi conduzida (Bicudo \& Miarka, 2015), e que se deu a partir do levantamento dos trabalhos publicados nos anais do SIPEM, ENEM, CIEM e SIPEMAT, realizados entre os anos de 2012 a 2018. O foco na Educação de Jovens e Adultos justifica-se pelo interesse acadêmico dos pesquisadores por essa modalidade de ensino, além da relevância desse estudo pauta-se em apresentar possíveis insights sobre o ensino e aprendizagem da Educação Estatística nessa etapa de ensino.

Para a produção dos dados, acessamos por meio do endereço eletrônico a página de cada evento. Como descritor, para localizar os trabalhos selecionados, foi utilizado o termo "Educação Estatística". Em seguida, passamos para a leitura atenciosa do título, resumo e palavras-chave. Dessa leitura, encontramos um universo de 172 trabalhos no campo da 
Educação Estatística. Esse exercício permitiu encontrarmos um universo de 10 (dez) trabalhos que passaram a constituir nosso corpus de pesquisa, pois se mostravam convergentes a nossa interrogação. Foram excluídos os trabalhos que não apresentaram explicitamente a temática da Educação Estatística no título ou no resumo, bem como trabalhos que abordavam a Educação Estatística, porém com foco diferente da Educação de Jovens e Adultos.

Com o intuito de organizar os trabalhos selecionados, realizamos um fichamento arquivado em uma planilha eletrônica, contendo as seguintes informações: edição do evento, ano do evento, autor, título dos trabalhos, instituição de origem, instrumentos de produção de dados, método da pesquisa, base teórica, modalidade de pesquisa (individual, em coautoria, em grupo, institucional, interinstitucional) e abrangência (local, estadual, nacional, internacional). Informações que consideramos importantes para nos auxiliarem nas análises dos trabalhos mapeados.

Por fim, realizamos a leitura integral de todos os trabalhos, orientando-nos pela interrogação de pesquisa: $\mathrm{O}$ que se mostra sobre a pesquisa em Educação Estatística na Educação de Jovens e Adultos publicadas nos anais do SIPEM, ENEM, CIEM e SIPEMAT? Objetivamos, portanto, delimitar o material de análise e aprofundar as reflexões acerca daquilo que se mostra do foco "Educação Estatística na Educação de Jovens e Adultos". Na tabela 1, a seguir, estão resumidos os números de trabalhos publicados no Grupo de Trabalho GT (12) e os trabalhos selecionados para a nossa análise.

Tabela 1 - Seleção de trabalhos para compor a metapesquisa

\begin{tabular}{llcc}
\hline Edição & $\mathbf{N}^{\mathbf{0}}$ & $\mathbf{N}^{\mathbf{0}}$ de trabalhos publicados no GT12 & $\mathbf{N}^{\mathbf{0}}$ trabalhos selecionados \\
\hline V SIPEM & 012 & 4 & $*$ \\
VI SIPEM & 015 & 4 & $*$ \\
VII SIPEM & 018 & 17 & $*$ \\
X ENEM & 010 & 23 & 2 \\
XI ENEM & 013 & 33 & 2 \\
XII ENEM & 016 & 20 & $*$ \\
VI CIEM & 013 & 10 & 1 \\
VII CIEM & 017 & 22 & 2 \\
SIPEMAT & 006 & 10 & $*$ \\
II SIPEMAT & 008 & 11 & $*$ \\
III SIPEMAT & 012 & 12 & 1 \\
V SIPEMAT & 018 & 6 & 2 \\
\hline
\end{tabular}

Fonte: Elaborado pelos autores

Adotamos como técnica de estudo a Análise de Conteúdo que, segundo Oliveira et al. (2003, p. 2), constitui-se como um "instrumento de análise interpretativa", uma vez que permite "descrever e interpretar o conteúdo de toda classe de documentos e textos [...] conduzindo a descrições sistemáticas [que ajudam] a atingir uma compreensão de seus significados num nível 
que vai além de uma leitura comum" (Moraes, 1999, p.1, inserção nossa). Em síntese, a Análise de Conteúdo “[...] se configura como um procedimento confiável para atingir as linhas mestras de um texto" (Oliveira et al., 2003, p.16).

\section{Apresentação E Discussão Dos Trabalhos}

Com base nessa técnica de estudo, dez artigos constituem efetivamente a metapesquisa de Educação Estatística na Educação de Jovens e Adultos. O Quadro 1 apresenta os artigos selecionados para este estudo.

Quadro 1 - Trabalhos selecionados

\begin{tabular}{|c|c|c|c|c|}
\hline Código & Edição & $\mathbf{N}^{\mathbf{0}}$ & Autor(es) & Título \\
\hline A1 & X ENEM & 2010 & $\begin{array}{l}\text { Izauriana Borges Lima; Ana } \\
\text { Coêlho Vieira Selva }\end{array}$ & $\begin{array}{l}\text { Investigando o desempenho de Jovens e } \\
\text { Adultos na construção e interpretação } \\
\text { de gráficos. }\end{array}$ \\
\hline A2 & X ENEM & 2010 & $\begin{array}{l}\text { Keli Cristina Conti; Dione } \\
\text { Lucchesi de Carvalho }\end{array}$ & $\begin{array}{l}\text { A construção de tabelas em aulas de } \\
\text { estatística na Educação de Jovens e } \\
\text { Adultos. }\end{array}$ \\
\hline A3 & XI ENEM & 2013 & $\begin{array}{c}\text { Lisiane Jaques Rodrigues } \\
\text { Scherwenske; Carla Gonçalves } \\
\text { Rodrigues }\end{array}$ & $\begin{array}{l}\text { A pesquisa eleitoral e a aprendizagem } \\
\text { de estatística: uma intervenção na } \\
\text { Educação de Jovens e Adultos. }\end{array}$ \\
\hline A4 & XI ENEM & 2013 & $\begin{array}{l}\text { Carlos Eduardo Ribeiro; Amari } \\
\text { Goulart }\end{array}$ & $\begin{array}{l}\text { O ensino de probabilidade por meio de } \\
\text { jogos na Educação de Jovens e Adultos }\end{array}$ \\
\hline A5 & VI CIEM & 2013 & Reinaldo Feio Lima; Lori Viali & $\begin{array}{c}\text { Os registros semióticos mobilizados por } \\
\text { alunos da EJA na interpretação de } \\
\text { dados em representações tabulares e } \\
\text { gráficas. }\end{array}$ \\
\hline A6 & VII CIEM & 2017 & Reinaldo Feio Lima & $\begin{array}{l}\text { Educação Estatística com base num } \\
\text { ciclo investigativo na Educação de } \\
\text { Jovens e Adultos. }\end{array}$ \\
\hline A7 & VII CIEM & 2017 & $\begin{array}{l}\text { Ewellen Tenorio de Lima; Rute } \\
\text { Elizabete de Souza Rosa Borba }\end{array}$ & $\begin{array}{l}\text { A determinação de espaços amostrais na } \\
\text { resolução de problemas combinatórios } \\
\text { na EJA }\end{array}$ \\
\hline A8 & III SIPEMAT & 2012 & $\begin{array}{l}\text { Izauriana Borges Lima; Ana } \\
\text { Coêlho Vieira Selva }\end{array}$ & $\begin{array}{l}\text { Interpretar e construir gráficos: qual é a } \\
\text { relação entre estas atividades? }\end{array}$ \\
\hline A9 & V SIPEMAT & 2018 & Reinaldo Feio Lima & $\begin{array}{l}\text { Desempenho de estudantes da EJA na } \\
\text { leitura, interpretação e construção de } \\
\text { gráficos e tabelas. }\end{array}$ \\
\hline A10 & V SIPEMAT & 2018 & $\begin{array}{l}\text { Cassio Cristiano Giordano; } \\
\text { Anderson Anzai dos Santos; } \\
\text { Cileda de Queiroz e Silva } \\
\text { Coutinho }\end{array}$ & $\begin{array}{c}\text { Estatística na Educação De Jovens e } \\
\text { Adultos: uma abordagem por meio de } \\
\text { projetos }\end{array}$ \\
\hline
\end{tabular}

Fonte: Elaborado pelos autores

Para procedermos com um exercício de apresentação e discussão dos dados, consideramos relevante na metapesquisa que as informações ou dados sejam organizados de maneira coerente e clara, uma vez que essa organização do material permite uma análise interpretativa mais cuidadosa, pois "esse processo é trabalhoso e meticuloso, [...] implica 
múltiplas leituras do material disponível, tentando nele buscar unidades de significado ou, então, padrões e regularidades para, depois, agrupá-los em categorias" (Fiorentini; Lorenzato, 2007, p.133).

A partir dessa informação começamos o movimento de análise e interpretação olhando atentamente para os trabalhos selecionados, para percebermos regularidades e padrões nos trabalhos. Essas regularidades serviram para que organizássemos essas informações em categorias. Para tanto, nos apropriamos do significado de categorização de Fiorentini e Lorenzato (2007, p.134) enquanto "um processo de classificação de informações em categorias, isto é, em classes ou conjuntos que contenham elementos ou características comuns, abrangendo todas as informações obtidas".

Dessa maneira, estabeleceram-se para análise seis categorias a priori para estudo. São elas: (a) sobre as instituições; (b) sobre autorias; (c) método e instrumentos de produção de dados; (d) sobre os aportes teóricos; (e) conteúdo abordado e; (f) o papel do estudante da EJA na pesquisa. Em seguida, efetuamos uma descrição e buscamos estabelecer uma síntese descritiva daquilo que cada categoria explicita e a interpretamos a partir daquilo que a categoria mostra como significativo na compreensão sobre as pesquisas em Educação Estatística com foco na Educação de Jovens e Adultos, os quais serão apresentados os principais pontos emergentes desta análise a seguir.

A categoria C1 - Sobre as instituições - apresenta as diferentes instituições de ensino onde as pesquisas têm sido desenvolvidas. Os trabalhos selecionados nos permitiram identificar diferentes regiões do país como: Norte, Nordeste, Sul e Sudeste, o que nos leva a inferir que, de fato, a Educação Estatística vem ganhando espaço no cenário educacional brasileiro [e para isso observamos o número de trabalho publicado nas edições do evento], seja público ou privado o ambiente acadêmico de pesquisa.

Por meio de um levantamento quantitativo simples é possível notar que a Região Sudeste é mais expressiva no que tange ao número de instituições de nível superior vinculadas aos trabalhos selecionados, totalizando três instituições, das quais duas são públicas e uma privada. Na sequência a região Sul contabiliza duas instituições, sendo uma pública e outra privada. Já as Regiões Norte e Nordeste, contam com duas instituições sendo todas públicas.

Em suma, essa categoria nos permite inferir que o desenvolvimento da pesquisa em Educação Estatística no país vem crescendo gradativamente. Destacamos que essa primeira interpretação se deu exclusivamente sobre as instituições de vínculo dos autores representadas nos trabalhos selecionados. 
Na sequência, a categoria C2 - Sobre autorias - indica a presença de dezenove autores para o total de dez trabalhos. Desse modo, destaca-se que a maioria dos trabalhos são desenvolvidos em coautoria, outros dois individualmente, (A6 e A9) e um trabalho escrito em trio (A10).

Outro dado que emerge de nossa análise, além da predominância de artigos desenvolvidos em coautoria, é que essa coautoria na sua maioria são membros conhecidos do GT12 e que os autores dos trabalhos selecionados são mestrandos ou doutorandos e que a coautoria se deu entre orientador e orientando (Araújo, 2009). No entanto, ressaltamos a ausência de trabalhos desenvolvidos em parceria interinstitucionais.

Na categoria C3 - Sobre método e instrumentos de produção de dados - procuramos descrever o método empregado nas pesquisas dos trabalhos analisados. Os trabalhos em sua maioria assumiram o método qualitativo de pesquisa, tendo apenas dois trabalhos com enfoque ao método misto (A1 e A7). Reconhecemos que a totalidade dos trabalhos se utiliza de método qualitativo de pesquisa, apresentam certa preocupação com o traçar metodológico e, nessa direção, concordamos com Araújo (2009, p. 12), quando aponta uma necessidade de “[...] aprender com as pesquisas já realizadas no Brasil e no exterior, por meio de uma ampla revisão da literatura, a fim de propor pesquisas inovadoras, que tragam efetivos avanços, e que primem pelo rigor metodológico".

Por outro lado, observamos que apenas uma minoria dos trabalhos selecionados explicitou o método utilizado no resumo. Isso nos sinaliza que houve, por parte desses pesquisadores, um cuidado na construção dos resumos e na reunião das informações necessárias para caracterização da pesquisa (Kluber, 2015). A maioria dos trabalhos selecionados não explicita no resumo que abordagem de pesquisa utilizou. Alguns trabalhos fazem essa apresentação do método dentro do texto, alguns de forma superficial, e outros em uma seção específica.

Quanto aos instrumentos utilizados para a produção de dados ocorreu principalmente por meio de teste diagnóstico ora articulada à entrevista ou intervenção. Nos artigos A1, A8 e A9, os autores desenvolveram suas pesquisas utilizando o instrumento teste diagnóstico com o intuito de compreender o desempenho de estudantes em atividades de construção e interpretação de gráficos e tabelas. Já nos artigos A5 e A6, os autores utilizaram um duplo instrumento de produção de dados, por um lado foi utilizado um teste diagnóstico prévio à realização da tarefa matemática (sem intervenção), e por outro, uma intervenção participativa.

$\mathrm{O}$ artigo A4 foi o único que utilizou uma sequência didática que versa sobre a introdução 
de conceitos básicos de probabilidade por meio de jogos para auxiliar na aprendizagem. Já nos artigos A2, A3 e A10 se embasam pelo uso projeto para a introdução da Educação Estatística, no que se refere à elaboração, leitura e interpretação de textos, tabelas e gráficos, bem como na mobilização de conhecimentos estatísticos para enfrentar os desafios em seu cotidiano (Batanero \& Díaz, 2011; Giordano \& Silva, 2017).

As pesquisas revisitadas, até aqui, sugerem que os métodos abordados nos trabalhos encontrados são, em sua maioria, testes diagnósticos elaborados pelos pesquisadores que se apropriam ao nível de escolarização, análise dos registros dos estudantes, seguida de uma intervenção e entrevistas clínicas.

Na categoria C4 - Sobre os aportes teóricos - buscamos analisar as correntes teóricas utilizadas pelos pesquisadores em suas investigações. Destacam-se, na análise dos trabalhos, a Teoria Registro de Representação Semiótica, presente em dois trabalhos publicados (A5 e A9), seguida da Teoria das Situações Didáticas (A4), Teoria dos Campos Conceituais (A7), Teoria de Vygostsky (A3) e os estudos de Gal (2002) e Garfield (2002), presente em quatro trabalhos (A1, A2, A6 e A10). Um dos artigos, A8, não apresenta uma base teórica acerca de EE para analisar as atividades de interpretação e construção de gráficos por estudantes da EJA. Alguns artigos analisados não apresentam fundamentação no que se refere à EE, embora tratem desta temática. De um modo geral, os artigos analisados demostram uma preocupação com a base teórica e análise dos dados.

Nos artigos A5 e A9, os autores usaram como suporte teórico a Teoria dos Registros de Representação Semiótica de Duval (2003), para identificar e compreender quais os registros de representação semiótica são mobilizados pelos estudantes da EJA ao resolverem testes envolvendo leitura e interpretação de tabelas e gráficos. No artigo A4 foi utilizado o referencial teórico da Teoria das Situações Didáticas para analisar os resultados de uma sequência didática que versa sobre a introdução de conceitos básicos de probabilidade por meio de jogos na EJA.

No artigo A7 utilizam-se da Teoria dos Campos Conceituais (Vergnaud, 1986, 1996) para investigar as contribuições que a exploração de situações-problema, referentes à Combinatória, traz para o raciocínio probabilístico na Educação de Jovens e Adultos e viceversa. Já o artigo A3 foi embasado na Teoria Interacionista de Vygotsky, com objetivo de testar uma hipótese curricular que contribuísse para a aprendizagem dos conceitos estatísticos a partir da temática do Processo Eleitoral.

De um modo geral, os artigos apresentam aportes teóricos consistentes ao seu tema de investigação, mas apresentam ausência de uma seção que discuta fundamentalmente a EE. 
Na categoria C5 - Conteúdos presente na pesquisa -, em relação aos conteúdos abordados pelos trabalhos aqui analisadas, percebemos que, em geral, os trabalhos versam sobre o bloco de conteúdos denominado "Tratamento da Informação", o qual se refere aos estudos sobre as noções de Estatística, Probabilidade e de Combinatória (A1, A2, A5, A6, A8 e A9), seguido sobre o ensino de Estatística (A3 e A10) e Conceitos Básicos de Combinatória e Probabilidade (A4 e A7). Nessa direção, nos reportamos a pesquisas, como a de Santos (2015), que defende o ensino articulado de Combinatória, Probabilidade e Estatística como forma de possibilitar o desenvolvimento de conceitos com significação desde a Educação Infantil.

A nosso ver, esta categoria nos permitiu inferir que a Educação Estatística na EJA recobre uma variedade de conteúdos matemáticos previstos no currículo de Matemática na Educação Básica. Isto é, os diversos temas abordados nos trabalhos selecionados ensejaram, em maior ou menor o grau, a revisão ou a apresentação de conteúdos que dessem conta de explicar os problemas decorrentes dos temas investigados. Nessa perspectiva, compreendemos que a Educação Estatística é mais uma proposta pedagógica para a Educação de Jovens e Adultos, contemplando os conteúdos necessários à escola e ao exercício de suas atividades cotidianas, sejam elas de âmbito profissional ou pessoal (Kluber, Mutti \& Silva, 2015).

Entretanto, enfatizamos que existe uma lacuna, nos artigos analisados, em relação à ausência de trabalhos voltados à Formação de Professores que ensinam Matemática, seja na Formação Inicial ou Continuada, ou propostas de investigações que busque identificar e oferecer subsídios aos professores para que eles possam proporcionar melhoria no processo de ensino e aprendizagem.

Quando olhamos para as produções em revistas científicas, com foco na formação de professores, encontramos os estudos de Batanero (2013) com foco na Didática da Estatística na formação de professores; Rocha, Lima e Borba (2016) conhecimentos pedagógicos para ensinar combinatória; Estevam e Cyrino (2016) letramento estatístico em contextos colaborativos; Cazorla (2017) ensino dos conteúdos conceituais e procedimentais de Estatística na formação dos professores e; Conti (2018) em contextos em Comunidades de Prática.

Esses pesquisadores, apresentados anteriormente, nos indicam caminhos e discutem abordagens em Educação Estatística acerca dos saberes no contexto da formação de professores que ensinam Matemática. Para Souza (2016), é fundamental que os professores estejam acostumados a lidar com essa abordagem desde sua formação inicial, isto é, o professor precisa estar preparado para engajar os estudantes em situações que envolvam problemas probabilísticos, combinatórios ou na formulação e na resolução de problemas que possam ser 
respondidos por meio da coleta, organização e análise de dados e da interpretação dos resultados (Estevam \& Cyrino, 2016).

Da mesma forma, também observamos uma carência de proposições didáticas que viessem a subsidiar professores que ensinam Matemática em relação ao como e ao porquê da relevância de trabalhar conceitos Probabilístico, Combinatórios e Estatísticos na sala de aula da EJA. Nesse sentido, destacamos a necessidade de refletir sobre a presença da Educação Estatística na formação inicial de professores que ensinam Matemática na EJA, no que diz respeito não só aos ambientes para a formação, mas também, as propostas de formação com Educação Estatística (Silva, Curri \& Schimiguel, 2017).

As pesquisas revisitadas, até aqui, sugerem que inúmeros sentidos podem ser desencadeados pelo discurso intradisciplinar na aprendizagem de temas envolvendo a Educação Estatística, por meio da relação entre os conteúdos da mesma disciplina, neste caso, a Matemática (Bernstein, 2000). Os trabalhos selecionados frequentemente expõem um sentido de que o discurso intradisciplinar favoreceu um efeito de alfabetização estatística. Os temas utilizados versam principalmente sobre questões envolvendo projeto ou teste diagnóstico seguida de uma intervenção de ensino. O envolvimento com esse tipo de proposta pedagógica favoreceu a imaginação, criatividade, conscientização e curiosidade dos estudantes sobre os conteúdos matemáticos. De modo geral, os estudos identificados mostram que os estudantes da EJA são capazes de desenvolverem o raciocínio combinatório, probabilísticos e estatísticos antes mesmo da sua introdução formal e as estratégias apresentadas por eles podem servir como base para intervenções mais próximas da sua forma de pensar (Assis, 2013; Lima; Miranda, 2013).

Outros aspectos evidenciados desses trabalhos geraram a categoria C6 - Sobre o papel do estudante da EJA na pesquisa - que revela o papel dos estudantes no desenvolvimento das tarefas matemáticas na perspectiva da Educação Estatística. Ela revelou que os estudantes desenvolveram atividades em grupos, duplas e individuais e desempenharam funções diversas. A pesquisa mostrou que os estudantes, de modo geral, demonstraram interesses em participar, pesquisar e construir situações que necessitavam de conhecimentos matemáticos para obterem respostas aos estudos de probabilidade, combinatória e estatística, tornando-se corresponsável por sua aprendizagem, por se tornar participante do processo de investigação.

Cabe aqui salientarmos também que as propostas de intervenções e projeto de pesquisa alcançaram importantes resultados para o ensino da Educação Estatística. Os estudos (A2, A3 e A10) têm em comum o trabalho com projetos como ponto de partida para a aprendizagem da 
Estatística. Conti e Carvalho (2010), Scherwenske e Rodrigues (2010) e Giordano, Santos e Coutinho (2018) deram destaque a uma abordagem por meio de projetos para a aprendizagem de conceitos estatísticos, em que o estudante assume uma postura mais participativa, na qual ele resolve problemas desenvolve projetos e, com isto, cria oportunidades para a construção de conhecimento (VALENTE, 2014). Os resultados desses três estudos indicaram que essa abordagem contribuiu para a aprendizagem acerca dos conceitos estatísticos, entre alunos de EJA, tanto em seus elementos cognitivos, mobilizando conhecimentos de contexto, de língua materna, de matemática, de estatística e competência para elaborar questões significativas, quanto nos elementos afetivos, que envolvem crenças, atitudes e postura crítica, assim como se percebeu a importância de criarem-se hipóteses curriculares diferenciadas, tendo em vista que, o processo em busca da aprendizagem com significado não pode seguir um padrão.

Outro aspecto importante em algumas das pesquisas são as intervenções que apontam para um ensino que venha a romper a perspectiva tradicional no qual se trabalham apenas com fórmulas. Por exemplo, Ribeiro e Goulart (2013) trabalharam com uma sequência que versa sobre a introdução de conceitos básicos de probabilidade por meio de jogos. Entre os resultados destacaram a relevância do uso de jogos para romper com o método tradicional de ensino e que no aspecto geral a sequência didática proporcionou desenvolver a percepção do acaso e a ideia de experimento aleatório.

Em suma, todas as pesquisas de intervenção constaram em seu método de pré-teste, intervenção e pós-teste, obtendo resultados significativos para o desenvolvimento do conceito pesquisado. Porciúncula e Samá (2015) indicam a satisfação dos estudantes quando estudam algo de seu interesse e o quanto isso influencia sua aprendizagem. Enfatizamos, a partir das pesquisas analisadas, que o uso de projetos no ensino de Estatística pode contribuir efetivamente para que os estudantes desenvolvam seu letramento estatístico ${ }^{1}$. Entre outros benefícios, ler com mais naturalidade gráficos e tabelas e informações veiculadas pela mídia como aponta Barberino e Magalhães (2016).

Os trabalhos selecionados também apontaram que, dependendo da postura assumida, a Educação Estatística pode desencadear uma formação crítica e reflexiva dos estudantes, os despertando para a aprendizagem. Atribuindo ao estudante o papel de protagonista, em tal processo, permite o desenvolvimento de habilidades e competências fundamentais para a promoção de sua autonomia intelectual e social. Tal aprendizagem está alicerçada no princípio

\footnotetext{
${ }^{1}$ Compreendemos o termo letramento estatístico como a capacidade de ler, interpretar e analisar criticamente as informações estatísticas, tais como gráficos, tabelas e análises de pesquisas (GAL, 2002).
} 
freiriano de autonomia, de protagonismo discente (Da Silva Pinto et al, 2014). Por fim, esses resultados representam o campo de potencialidades que a Educação Estatística com foco na Educação de Jovens e Adultos oferece aos estudos da Educação Matemática, quando diz respeito ao ensino, a aprendizagem e a pesquisa científica nessa região de inquérito.

\section{Considerações Finais}

Neste artigo teórico, a partir da questão e objetivo apresentado, foi realizada uma identificação e compreensão do como se apresentam a produção da pesquisa em Educação Estatística que tem como foco o ensino aprendizagem na Educação de Jovens e Adultos, socializada por ocasião nos anais dos eventos SIPEM, ENEM, CIEM e SIPEMAT, no período de 2002 a 2018. Foram encontrados 10 artigos de pesquisas diagnósticas ou de intervenção com estudantes. A metapesquisa empreendida, como bem expressa o prefixo "meta", permitiu transcender, ir além, refletir criticamente sobre as pesquisas realizadas no âmbito da EE na EJA.

A pesquisa por nós empreendida, sob a questão: O que se mostra sobre a pesquisa em Educação Estatística na Educação de Jovens e Adultos publicadas nos anais do SIPEM, ENEM, CIEM e SIPEMAT? Fez emergir seis categorias a priori para estudo: (a) sobre as instituições; (b) sobre autorias; (c) método e instrumentos de produção de dados; (d) sobre os aportes teóricos; (e) conteúdo abordado e; (f) o papel do estudante da EJA na pesquisa. Tais categorias corroboram a busca por respostas pera essa questão.

Essa metapesquisa avançou sistematizando as principais características/contribuições dos dez trabalhos analisados e também desafios a serem enfrentados para a inserção da Educação Estatística no contexto da Educação de Jovens Adultos e, na/para formação de professores que ensinam Matemática nesta modalidade de ensino. Ela apresenta para a comunidade de pesquisadores brasileiros, em especial ao Grupo de Trabalho sobre Ensino de Probabilidade e Estatística (GT 12) da Sociedade Brasileira de Educação Matemática (SBEM), que vem crescendo como área de pesquisa, ainda que de modo indicativo e não prescritivo, possibilidades de crescimento teórico e prático quando nos centramos na Educação Estatística de Jovens e Adultos. Para tal, enquanto grupo de trabalho da SBEM que vem se constituindo e se fortalecendo ao longo dos anos, a Educação Estatística utiliza-se de recursos teóricometodológicos de outras áreas, como Educação Matemática, Psicologia, Pedagogia, Filosofia e Matemática, além da própria Estatística.

Em suma, daquilo que foi apresentado e discutido pelas categorias a priori sobre o 
campo de pesquisa da Educação Estatística com foco na Educação de Jovens e Adultos, afirmamos que os textos aqui analisados revelam a variedade de temáticas abordadas nesses trabalhos, direcionando-se para uma Educação Estatística para Estudantes (EEpE). Podemos, assim, dizer que são investigações em que estudante assume o papel de protagonista em sala de aula como um ambiente formador. Isso revela que as pesquisas em EE também estão preocupadas com as especificidades da sala de aula. Frente a essa compreensão revela-se que a pesquisa em Educação Estatística continua buscando e apresentando argumentos, novas metodologias e propostas didáticas de ensino que justifiquem a sua utilização em sala aula.

Percebemos, a partir da análise realizada e como apresentamos em outro momento do texto, que existe uma lacuna nos artigos analisados em relação à ausência de trabalhos voltados à Formação de Professores que ensinam Matemática, seja na Formação Inicial ou Continuada, ou propostas de investigações que busque identificar e oferecer subsídios aos professores para que eles possam proporcionar melhoria no processo de ensino e aprendizagem. Deixamos, portanto, estas possíveis sugestões para o campo de pesquisa.

Por fim, ainda enquanto compromisso com o campo de pesquisa, pretendemos dar continuidade na análise e classificação dos artigos selecionados, lançando novos olhares aos livros e as dissertações e teses defendidas, para que se possa ter uma análise mais global do desenvolvimento do campo de pesquisa da Educação Estatística.

\section{Agradecimentos}

Ainda que não seja responsável pelas posições adotadas neste artigo, nossos agradecimentos a Professora $\mathrm{Dr}^{\mathrm{a}}$. Andreia Maria Pereira de Oliveira.

\section{Referências}

Andre, M. (2010). Formação de professores: a constituição de um campo de estudos. Educação PUCRS. http://revistaseletronicas.pucrs.br/ojs/index.php/faced/article/view/8075

André, M. (2001). Pesquisa em educação: buscando rigor e qualidade. Caderno de Pesquisa. [online]. Recuperado de http://www.scielo.br/pdf/cp/n113/a03n113.pdf

Araújo, J. L. (2009, outubro) Pesquisas sobre modelagem em eventos científicos recentes de educação matemática no Brasil. In: Anais do Seminário Internacional de Pesquisa em Educação Matemática(pp. 1-15), Taguatinga, DF: Sociedade Brasileira de Educação Matemática. Recuperado de http://www.sbem.com.br/sipem/files/resumos.pdf 
Assis, A. (2013, julho). Formação docente: a compreensão da combinatória a partir dos significados, invariantes e representações simbólicas. In: Anais do XI Encontro Nacional de Educação Matemática(pp. 1-15). Curitiba, PR: Sociedade Brasileira de Educação Matemática. Recuperado de http://sbem.iuri0094.hospedagemdesites.ws/anais/XIENEM/trabalhos_1.html

Barberino, M. R. B. \& Magalhães, M. N. (2016). Uma proposta para desenvolver o letramento estatístico através de projetos. Vidya. Recuperado de https://periodicos.ufn.edu.br/index.php/VIDYA/article/view/1821/1745

Barbosa, J. C. (2007, novembro). Sobre a pesquisa em Modelagem Matemática no Brasil. In $I V$ Conferência Nacional sobre Modelagem na Educação Matemática(pp. 82-103). Ouro Preto, MG: Sociedade Brasileira de Educação Matemática. CD-ROM

Batanero, C. (2013, junio). Sentido estadístico: componentes y desarrollo. In: Jornadas virtuales en didáctica de la estadística, probabilidad y combinatória(p. 55-61), Granada, Espanha. Actas. Recuperado de http://www.ugr.es/ jmcontreras/pages/Investigacion/Actas\%20jornadas.pdf

Batanero, C., \& DÍAZ, C. (2011). Estadística con proyectos. Granada (España), Universidad de Granada.

Bicudo, M. A., \& Miarka R. (2015). Metodologia de Pesquisa em Filosofia da Educação Matemática- Perspectivas da Educação Matemática. Recuperado de http://seer.ufms.br/index.php/pedmat/article/view/1439/964

Bicudo, M. A. V. A. (2012). Pesquisa em educação matemática: a prevalência da abordagem qualitativa. Revista Brasileira de Ensino de Ciência e Tecnologia. Recuperado de https://periodicos.utfpr.edu.br/rbect/article/view/1185

Bicudo, M. A. V., \& Monteiro, P. R. (2011). Um Exercício Filosófico sobre a Pesquisa em Educação Matemática no Brasil. Bolema. Recuperado de http://www.redalyc.org/pdf/2912/291223514012.pdf

Bogdan, R., \& Biklen, S. (2010). Investigação qualitativa em Educação: fundamentos, métodos e técnicas. In: Investigação qualitativa em educação. Portugal: Porto Editora.

Cazorla, I., Magina S., Gitiran, V., \& Guimarães, G. (2017). Estatística para os anos iniciais do ensino fundamental. Brasília. Sociedade Brasileira de Educação Matemática.

Conti, K. C. O. (2018). Estudo da estatística num contexto colaborativo: o gráfico de setores. REnCiMa. Recuperado de http://revistapos.cruzeirodosul.edu.br/index.php/rencima/article/view/1667/971

Conti, K. C., \& Carvalho, D. L. A. (2013, julho). Construção de tabelas em aulas de estatística na educação de jovens e adultos. In: Anais do X Encontro Nacional de Educação Matemática(pp.1-11). Curitiba, PR. Sociedade Brasileira de Educação Matemática. Recuperado de http://www.lematec.net.br/CDS/ENEM10/artigos/CC/T2_CC84.pdf 
Cury, H. N. (2013). Erros, Dificuldades e obstáculos em produções scritas de alunos e professores. In: Frota, M. C. R.; Bianchini, B. L.; Carvalho, A.F.T. Marcas da Educação Matemática no Ensino Superior. Campinas, SP: Papirus.

Da Silva Pinto, A. S., Bueno, M. R. P., Silva, M. A. F. A., Menezes, M. Z. S., \& Koehler, S. M. F. (2014). O Laboratório de Metodologias Inovadoras e sua pesquisa sobre o uso de metodologias ativas pelos cursos de licenciatura do UNISAL, Lorena-estendendo o conhecimento para além da sala de aula. Revista de Ciências da Educação. Recuperado de www.revista.unisal.br/ojs/index.php/educacao/article/view/288

Estevam, E.J.G., \& Cyrino, M.C.C.T. (2016). Comunidades de Prática como contexto para o desenvolvimento profissional docente em Educação Estatística. Educação Matemática Pesquisa. Recuperado de https://revistas.pucsp.br/emp/article/view/31485/21944

Fiorentini, D., \& Lorenzato, S. (2007). Investigação em Educação Matemática: percursos teóricos e metodológicos. São Paulo. Autores Associados.

Gal, I. (2002) Adult's statistical literacy: meanings, components, responsabilities. International Statistical Review. Recuperado de https://iaseweb.org/documents/intstatreview/02.Gal.pdf

Giordano, C. C., Santos, A. A. S., \& Coutinho, C. Q. S. (2018, junho). Estatística na educação de jovens e adultos: uma abordagem por meio de projetos. In: Anais do Simpósio Internacional de Pesquisa em Educação Matemática(pp.1-16). Belém, PA. Recuperado de https://www.researchgate.net/publication/329629162_estatistica_na_educacao_de_jovens _e_adultos_uma_abordagem_por_meio_de_projetos

Giordano, C. C., \& Silva, D. S. C. (2017). Metodologias ativas em educação matemática: a abordagem por meio de projetos na educação estatística. - Revista de Produção Discente em Educação Matemática. https://revistas.pucsp.br/pdemat/article/view/35422/24249

Kluber, T. E., Mutti, G. S. L., \& Silva, M. V. (2015). Modelagem matemática (MM) na educação de jovens e adultos (EJA): contribuições a partir de um metaestudo. Revista PerCursos. Recuperado http://www.periodicos.udesc.br/index.php/percursos/article/view/1984724616312015083/ pdf 28

Lima, E. T., \& Borba, R. E. S. R. A. (2017, outubro). Determinação de espaços amostrais na resolução de problemas combinatórios na EJA. In: Anais do Congresso Internacional de Ensino de Matemática(pp.1-14). Canoas, RS: ULBRA. Recuperado de http://www.conferencias.ulbra.br/index.php/ciem/vii/paper/viewFile/6683/3739

Lima, I. B. \& Selva, A. C. V. (2013, julho). Investigando o desempenho de jovens e adultos na construção e interpretação de gráficos. In: Anais do Encontro Nacional de Educação Matemática(pp.1-11). Curitiba, PR: Sociedade Brasileira de Educação Matemática. Recuperado de http://www.lematec.net.br/CDS/ENEM10/artigos/CC/T2_CC814.pdf

Lima, I. B., \& Selva, A. C. V. (2012, outubro). Interpretar e construir gráficos: qual é a relação entre estas atividades? In: Anais do Simpósio Internacional de Pesquisa em Educação 
Matemática(pp.1-14), Petrópolis, Rio de Janeiro: Sociedade Brasileira de Educação Matemática. Recuperado de http://www.sbembrasil.org.br/files/v sipem/?page=publications\&language $=\mathrm{br}$

Lima, T., \& Miranda, D. (2013, julho). O uso de diferentes registros de representação na resolução de problemas que envolvem análise combinatória. In: Anais do XI Encontro Nacional de Educação Matemática(pp. 1-13). Curitiba, PR: Sociedade Brasileira de Educação Matemática. Recuperado de http://sbem.iuri0094.hospedagemdesites.ws/anais/XIENEM/pdf/1707 428 ID.pdf

Lima, R. F., \& Viali, L. (2013, outubro). Os registros semióticos mobilizados por alunos da EJA na interpretação de dados em representações tabulares e gráficas. In: Anais do Congresso Internacional de Ensino de Matemática(pp.1-13). Canoas, RS: ULBRA. Recuperado de https://www.researchgate.net/publication/280446976_Os_registros_semioticos_mobilizad os_por_alunos da_EJA_na interpretacao de dados_em representacoes tabulares e gra ficas

Lima, R. F. (2017, outubro). Educação estatística com base num ciclo investigativo na Educação de Jovens e Adultos. In: Anais do Congresso Internacional de Ensino de Matemática(pp.114). Canoas, RS: ULBRA. Recuperado de http://www.conferencias.ulbra.br/index.php/ciem/vii/paper/viewFile/6452/3098

Lima, R. F. (2018, junho). Desempenho de estudantes da EJA na leitura, interpretação e construção de gráficos e tabelas. In: Anais do Simpósio Internacional De Pesquisa Em Educação Matemática(pp.1-14). Belém, PA. Recuperado de http://sipemat2018.sbempara.com.br/files/Anais_SIPEMATParte1.pdf

Lopes, T. B., Palma, R. C. D., \& Sá, P. F. (2018). Engenharia didática como metodologia de pesquisa nos projetos publicados no EBRAPEM (2014-2016). Educação Matemática Pesquisa. Recuperado de https://revistas.pucsp.br/emp/article/view/34925/pdf

Oliveira, E., Ens, R. T., Andrade, D. B. S. F., \& Muss, C. R. (2003). Análise de Conteúdo e Pesquisa na Área da Educação. Revista Diálogo Educacional. Recuperado de https://periodicos.pucpr.br/index.php/dialogoeducacional/article/view/6479

Moraes, R.(1999). Análise de conteúdo. Revista Educação. Recuperado de https://edisciplinas.usp.br/pluginfile.php/4125089/mod_resource/content/1/RoqueMoraes Analise\%20de\%20conteudo-1999.pdf

Pessoa, C., \& Silva, M. (2012, junho). Invariantes, generalização, sistematização e estratégias bem sucedidas: o ensino da combinatória no $9^{\circ}$ ano do Ensino Fundamental. In Anais do III Simpósio Internacional de Educação Matemática(pp. 1-15). Fortaleza, CE: Sociedade Brasileira de Educação Matemática. Recuperado de http://proativa.virtual.ufc.br/sipemat2012/apresentacoes.htm

Porciúncula. M., \& Samá, S. (2015). Projetos de Aprendizagem: uma proposta pedagógica para a Sala de Aula de Estatística. In Samá, S.; Porciúncula, M.(org.) Educação Estatística: ações e estratégias pedagógicas no Ensino Básico e Superior. Curitiba: CRV.

Ribeiro, C. E., \& Goulart, A. (2010, julho). O ensino de probabilidade por meio de jogos na 
Educação de Jovens e Adultos. In: Anais do X Encontro Nacional De Educação Matemática (pp.1-11). Salvador, BA: Sociedade Brasileira de Educação Matemática Recuperado de http://www.lematec.net.br/CDS/ENEM10/

Rocha, C.; Lima, A. P.; Borba, R. (2016). Conhecimentos de professores para ensinar Combinatória: currículo e documentos orientadores para os anos iniciais. EM TEIA Revista de Educação Matemática e Tecnológica Iberoamericana. Recuperado de https://periodicos.ufpe.br/revistas/emteia/article/view/3895/pdf

Santos, J. A. (2015) Produção de significações sobre combinatória e probabilidade numa sala de aula do $6^{\circ}$ ano do ensino fundamental a partir de uma prática problematizadora (Tese de Doutorado em Educação). Universidade São Francisco, São Paulo.

Scherwenske, L. J. R., \& Rodrigues, C. G. (2010, julho). A pesquisa eleitoral e a aprendizagem de estatística: uma intervenção na Educação de Jovens e Adultos. In: Anais de X Encontro Nacional de Educação Matemática(pp. 1-11) Salvador, BA: Sociedade Brasileira de Educação Matemática. Recuperado de http://www.lematec.net.br/CDS/ENEM10/

Silva, J. F. \& Curri, E.; Schimiguel, J. (2017). Um cenário sobre a pesquisa em Educação Estatística no Boletim de Educação Matemática - Bolema, de 2006 até 2015. Bolema. Recuperado de http://www.scielo.br/pdf/bolema/v31n58/0103-636X-bolema-31-580679.pdf

Silva, M. C., \& Pessoa, C. A. S. (2015). A combinatória: estado da arte em anais de eventos científicos nacionais e internacionais ocorridos no Brasil de 2009 a 2013. Educação Matemática Pesquisa. Recuperado de https://revistas.pucsp.br/emp/article/view/20665/pdf

Souza, A. C. (2013). O desenvolvimento profissional de educadoras da infância: uma aproximação à Educação Estatística. (Tese de Doutorado em Ensino de Ciências e Matemática). Universidade Cruzeiro do Sul, São Paulo.

Valente, J. A. (2014). Blended Learning e as mudanças no ensino superior: a proposta da sala de aula invertida. Educar em Revista. Recuperado de http://www.scielo.br/pdf/er/nspe4/0101-4358-er-esp-04-00079.pdf 